\title{
Development of fast switching photochromic coatings on transparent plastics and glass
}

\author{
M. Mennig*, K. Fries, M. Lindenstruth, H. Schmidt \\ Institut Fuer Neue Materialien, Saarbruecken, Germany
}

\begin{abstract}
A new photochromic organic-inorganic nanocomposite (Nanomer ${ }^{(0)}$ ) coating system has been developed. It is based on an epoxysilane as network former, an organic bisepoxide as spacer, an organic amine as thermal cross-linker and surface modified $\mathrm{SiO}_{2}$ nanoparticles as fillers. This coating system is compatible with different photochromic dyes, like oxazines and pyrans. Photochromic coatings with blue, yellow, red, green, violet and neutral tints were prepared on flat glass and PMMA substrates by dip coating and cured for $3 \mathrm{~h}$ at $100^{\circ} \mathrm{C}$. The photochromic coatings show the following properties: the transmittance changes reversibly between 80 and $20 \%$ with half fading times of about $2-20 \mathrm{~s}$ (comparable to the half fading time of the appropriate dye in ethanolic solution) after UV irradiation for $15 \mathrm{~s}$ with $5 \mathrm{~mW} / \mathrm{cm}^{2}$. The coatings show a scratch hardness of about $15 \mathrm{~g}$ (scratch test with Vickers indenter, coating thickness $10 \mu \mathrm{m}$ ). The addition of only 3 wt.\% $\mathrm{SiO}_{2}$ nanoparticles relative to epoxysilane increases the scratch resistance of the coatings to about $20 \mathrm{~g}$ without changing the fast kinetics of the incorporated photochromic dye(s). The long term stability of the photochromic dye(s) in the matrix system can be considerably improved by the introduction of additives like antioxidants, hindered amine light stabilizer (HALS) and UV stabilizers. The half lifetime (decay to $50 \%$ of the initial photochromic intensity) of a blue spirooxazine dye (Blue A) measured in a dry sun-test $\left(75 \mathrm{~mW} / \mathrm{cm}^{2}\right)$ could be increased from $20 \mathrm{~h}$ without any additive un to $200 \mathrm{~h}$ with an UV ahsorher (Tinuvin 327) as a stabilizer, which is assumed to be sufficient for ophthalmic applications.
\end{abstract}

Keywords: Photochromic coatings; Organic-inorganic hybrids

\section{Introduction}

Photochromism is the reversible colour change undergone by a compound under UV irradiation. The photochromic phenomenon is exhibited by two different classes of materials. Metal halides in melted special glasses are used as active materials for ophthalmic applications. In addition, organic dyes as spirooxazines, spiropyrans, chromenes, dihydroindolizines in organic polymers show a photochromic effect [1]. Inorganic photochromes offer only a limited colour range and are not suitable for treating organic polymeric substrates as there exists no coating material for such an application, but photochromic polymeric lenses have been obtained with organic photochromic dyes by thermal transfer technique (high processing costs) [2]. Ophthalmic polymer bulks with incorporated organic photochromes show switching times of about $100 \mathrm{~s}$ and more [2] and photochromic coatings on CR 39 (PPG) result in insufficient scratch hardness so that a multilayer is required [3].

In general, one may assume that a certain inner free \footnotetext{
223.

* Corresponding author. Tel.: +49-681-9300-394; fax: +49-681-9300-

E-mail address: mennig@inm-gmbh.de (M. Mennig)
}

volume of the host material would be required to avoid sterical hindering for the switching process. On the other hand, reactions of the photochromic dyes with moisture and oxygen from the environment, which lead to a decay of the photochromic activity [4] should be hindered by a minimum inner free volume of the host and for many practical applications a high scratch hardness of the photochromic material would be required. Using polymeric matrices for the photochromic effect, soft polymers are not applicable because of the poor mechanical properties and in hard polymers the switching behaviour of the photochromic dyes is hindered.

In order to fulfil these antagonistic demands, the following general approach of a nanocomposite material with ceramic nanoparticles in an organic-inorganic matrix was chosen for the development of photochromic coatings with a great variety of colours for glass and plastic substrates. The organic-inorganic matrix should have sufficient free volume for the switching process of the dyes in the soft spacing zones between the embedded ceramic nanoparticles, which increase the hermicity and the mechanical durability of the matrix.

As a base line of this development, a model system for photochromic coatings on glass based on 3-glycidyloxypro- 
pyl-trimethoxysilane (GPTS), bisphenole A (BPA) and methylimidazole (MI) $[3,5,6]$ was used. However, this system was only suitable for yellow pyran and blue spirooxazine dyes, but not suitable for violet spirooxazine and for coating plastic substrates (PMMA, PC) [7].

\section{Experimental}

For the preparytion of the photochromic coatings, $10.5 \mathrm{~g}$ GPTS (3-glycidoxypropyl-trimethoxysilane) pre-hydrolysate [8] as a network former and $2.5 \mathrm{~g}$ bis-(epoxycyclohexyl)-methylcarboxylate as a spacer were mixed at room temperature. After that, 0.06 mmole photochromic dye or 0.4 mmole of a dye mixture (neutral tint) were dissolved in the sol at room temperature. In sol systems with UV-stabilizers, the UV-stabilizer was added after dissolving the photochromic dye in the sol with a molar ratio of $1: 1$ relative to the dye. Then $1 \mathrm{~g}$ isophorondiamine was added as a crosslinker and stirred for a few minutes before the sol was filtered (1.0 $\mu \mathrm{m}$ PTFE syringe filter). The incorporated photochromic dyes and UV-stabilizers (UV-absorber, HALS, antioxidants) are all commercially available.

Coating systems with $\mathrm{SiO}_{2}$ nanoparticles were also prepared using a 1:1 molar mixture of $\left(\mathrm{GPTS} / \mathrm{SiO}_{2}\right)$ prehydrolysate $[5,6]$ and GPTS pre-hydrolysate instead of only GPTS pre-hydrolysate.

After dip coating of the sol systems onto glass and PMMA substrates, the coatings were thermally densified for $3 \mathrm{~h}$ at $100^{\circ} \mathrm{C}$. The adhesion of the coatings was measured by cross cut and tape test [9].

Two characteristic photochromic properties, darkening efficiency $\Delta A_{0}$ (difference between maximum absorbance during the photostationary state under UV irradiation and the minimum absorbance in the bleached state) and halffading time $t_{0.5}$ (time difference required for $\Delta A_{0}$ to decay to $50 \%$ of the initial value after cut-off of the UV source) of these coatings were measured with an UV-VIS spectrophotometer (HP 8453) using an external Xe-lamp (Osram XBO $450 \mathrm{~W} / 1)$ for irradiation $\left(5 \mathrm{~mW} / \mathrm{cm}^{2}\right)$ under isothermal condi-

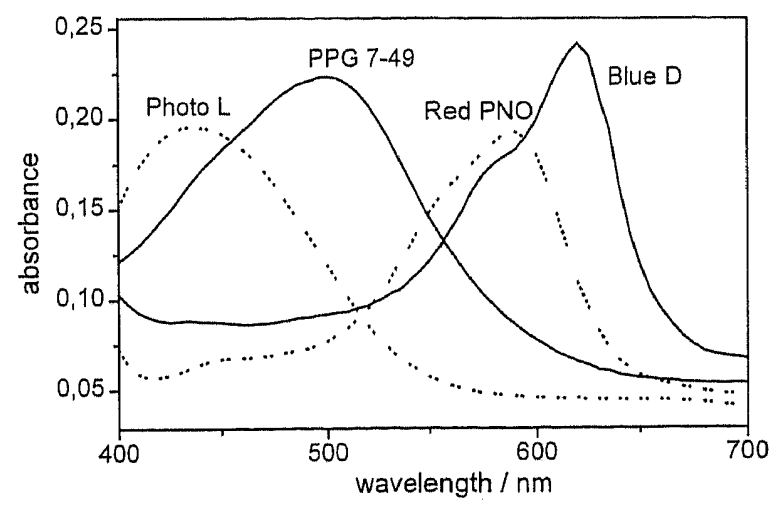

Fig. 1. Absorbance spectra of photochromic coated glass slides under UV irradiation $\left(5 \mathrm{~mW} / \mathrm{cm}^{2}\right.$ for $\left.15 \mathrm{~s}\right)$ with the photochromic dyes Blue D (blue), PPG 7-49 (red), Red PNO (violet) and Photo L (yellow).

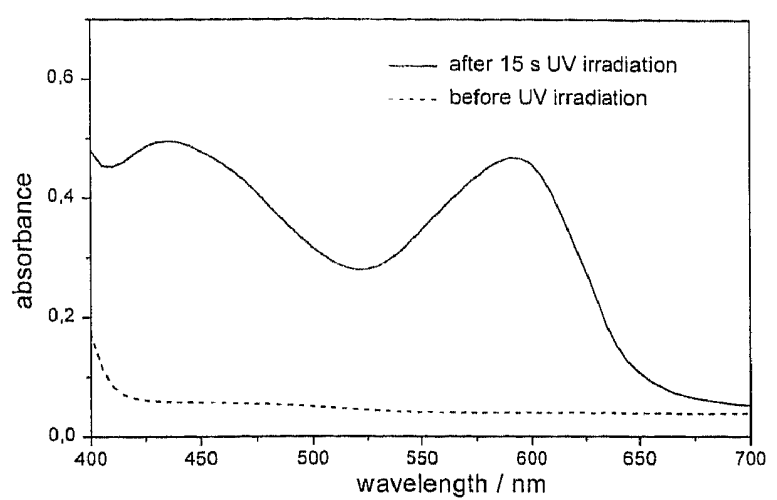

Fig. 2. Absorbance spectra of a neutral photochromic coating on PMMA with Red PNO + Photo L + Blue A (45:40:15 mole\%) before and after $\mathrm{UV}$ irradiation $\left(5 \mathrm{~mW} / \mathrm{cm}^{2}, 15 \mathrm{~s}\right)$.

tions (room temperature). Furthermore, the scratch hardness of different coatings was investigated using a Vickers indenter. The load, at which the first scratch could be detected in a microscope was taken as a measure for the scratch hardness. The UV stability of the coatings was investigated using a dry sun-test (Atlas CPS, $75 \mathrm{~mW} / \mathrm{cm}^{2}$, without cooling). From the decay of the photochromic intensity, the time was determined in which the initial value of $\Delta A_{0}$ was decreased to $50 \%$.

\section{Results}

The use of a bisepoxide as a spacer in the matrix system is assumed to be sufficient for the creation of high free pore volume so that the photochromic dyes are not hindered in their kinetic behaviour. Furthermore, the used amount of $\mathrm{SiO}_{2}$ nanoparticles ( $3 \mathrm{wt} . \%$ relative to the epoxysilane) increase the mechanical properties of the coating without changing the fast switching kinetic of the incorporated dyes.

By the experimental preparation as described above, transparent and colourless coatings containing different photochromes have been obtained. Fig. 1 shows four absorption spectra of coatings with different photochromic dyes under UV irradiation.

The different photochromic dyes were chosen to demonstrate the great variety of colours. As can be seen from Fig. 1, the photochromic colours of the activated coatings range from yellow (absorption maximum of the activated state $435 \mathrm{~nm}$ ) to blue (absorption maximum at $620 \mathrm{~nm}$ ). The coatings show good adhesion (measured by cross-cut and tape test [9]) on glass and also on PMMA.

Different photochromic dyes were mixed in order to obtain neutral tints in the coatings. Fig. 2 shows the absorbance spectra of a grey coloured photochromic coating on PMMA before and after UV irradiation.

As can be seen from Fig. 2, practically no absorption is obtained in the bleached state. After UV irradiation $(5 \mathrm{~mW} /$ $\mathrm{cm}^{2}, 15 \mathrm{~s}$ ) two absorption peaks at $435 \mathrm{~nm}$ (due to the absorption caused by Photo L) and at $595 \mathrm{~nm}$ (due to the 


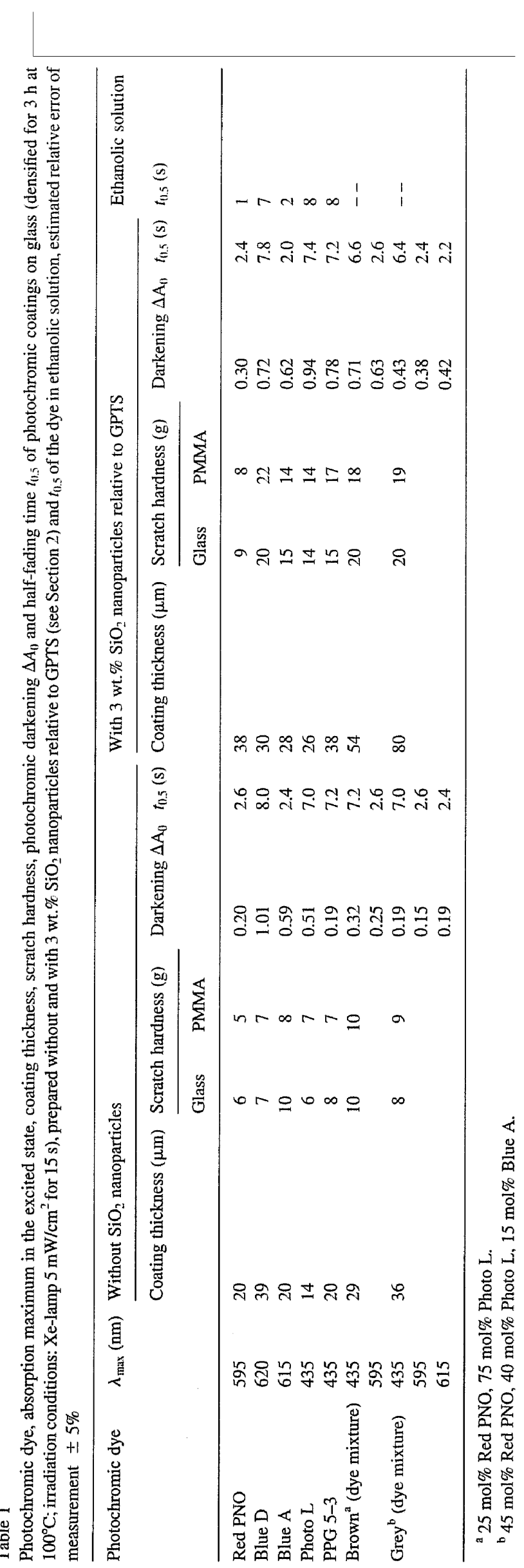


absorption caused by Red PNO) are clearly observed. The absorption caused by Blue $A$ (peak at about $615 \mathrm{~nm}$ ) is overlapped by the absorption caused by Red PNO. Although there is a minimum around $520 \mathrm{~nm}$, the coating appears neutral grey, because the sensitivity of the human eye is very high in this spectral range. In Table 1 the coating thickness, scratch hardness and the photochromic properties of the different photochromic coatings are summarized.

As can be seen from the reported $\Delta A_{0}$ values in Table 1 , the solubility of the dyes in the sol systems is sufficient to obtain intense colours in coatings with $20-40 \mu \mathrm{m}$ thickness under UV-irradiation $\left(5 \mathrm{~mW} / \mathrm{cm}^{2}\right)$ for $15 \mathrm{~s}$. The photochromic intensity of the coating depends on the molar coefficient of absorbance of the dye, but also on the coating thickness and on the dye concentration in the matrix. For a diluted system with passive, absorbing dyes, the absorbance is a linear function of the dye concentration and thickness of the layer (Lambert-Beer law). For active dyes like photochromic dyes, the relationship between absorbance, dye concentration and coating thickness has to be investigated in principle, because the absorbance of the exciting irradiation is also a function of the coating thickness (penetration depth of the UV light) and dye concentration. For this purpose, coatings with the blue spirooxazine dye (Blue A) in a concentration of $3 \times 10^{-2} \mathrm{~mole} / \mathrm{l}$ (close to the solubility limit for this dye in this system) and different thicknesses have been prepared and the saturation darkening was measured under standard conditions. The result is shown in Fig. 3.

Fig. 3 shows that the absorbance is increasing with the coating thickness and a saturation is obtained for thicknesses higher than $40 \mu \mathrm{m}$. This saturation can be attributed to the fact that the UV light, required for the excitation of the dye molecules, is strongly absorbed by the dye and therefore the intensity of the UV light is decreasing with increasing depth of the coating.

For the fading process of the darkened coatings to 70

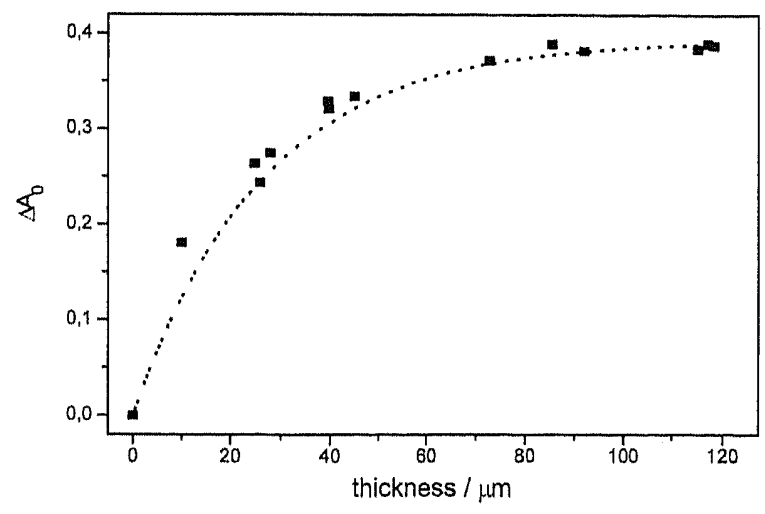

Fig. 3. Darkening $\Delta A_{1}$ of Nanomer ${ }^{\text {no }}$ coatings (without 3 wt.\% $\mathrm{SiO}_{2}$ nanoparticles, see Section 2) containing spirooxazine dye Blue $\mathrm{A}\left(3 \times 10^{2} \mathrm{~mole} /\right.$ 1) on glass (densified at $100^{\circ} \mathrm{C}$ for $3 \mathrm{~h}$ ) in dependence of the coating thickness (estimated relative error of measurement $\pm 5 \%$, the line is drawn as a guide for the eye).
$90 \%$ transmittance, half-fading times of 3 to 10 s have been obtained. In the last column of Table 1, the half-fading times of the appropriate dyes in alcoholic solution, determined with the same experimental set-up in a $1 \mathrm{~cm}$ cuvette, are given for comparison. The half-fading times of the dyes in the coatings do not differ significantly from those measured in alcohol. From these investigations, it can be concluded that the Nanomer coating matrix does not hinder sterically the photochromic switching process. Table 1 also shows that neutral tints (brown - grey) have been realized by mixing different photochromes with similar kinetic behaviours.

The scratch hardnesses of the uncoated substrates were determined to be $2 \mathrm{~g}$ for PMMA and $<1 \mathrm{~g}$ for glass. As can be seen from Table 1, the scratch hardness of the coating was in the range between 6 to $10 \mathrm{~g}$. There is no significant difference between the scratch hardness of coatings on glass and on PMMA within the error of measurement, estimated at $\pm 3 \mathrm{~g}$. The scratch hardness was further increased to the range between 9 to $20 \mathrm{~g}$ by the addition of the solvent free GPTS pre-hydrolysate containing the $\mathrm{SiO}_{2}$ nanoparticles [10]. By this, the solid content in the coating was only increased by $3 \mathrm{wt} \% \mathrm{SiO}_{2}$ relative to the GPTS content, which was kept constant. It is assumed that the strong increase of the scratch hardness cannot only be attributed to the rather small content of $\mathrm{SiO}_{2}$ nanoparticles but may be also due to the addition of the solvent free GPTS pre-hydrolysate [10]. It is replacing 50\% of the GPTS pre-hydrolysate prepared after [8] and it is assumed that the latter has a lower degree of condensation. However, appropriate experiments will have to be carried out in the future. The used scratch test with a Vicker's indenter leads to damages especially for soft but also for brittle surfaces like glass. A high scratch hardness can be attributed to a hard-elastic behaviour of the coating. However for more detailed information on the mechanical properties of the coatings (e.g. plastic and elastic contributions to deformation), further investigations will be required. Table 1 also shows that the bleaching kinetics are not affected at all by the incorporation of the solvent free GPTS pre-hydrolysate with the $\mathrm{SiO}_{2}$ nanoparticles.

This strengthens the hypothesis that photochromic materials with fast switching kinetics in combination with macroscopic hard properties can be realized by the nanocomposite approach.

The long term UV stability of photochromic dyes in solgel based coatings is one of the crucial points for practical application. The activated photochromic dye is easily degraded by $\mathrm{OH}$-attack, oxidation $\left(\mathrm{O}_{2}\right.$ from air, redox reaction with polyvalent components) or degradation by absorption of (hard) UV light [4]. In order to improve the photostability of the photochromic coating, different additives like antioxidants, HALS (Hindered Amine Light Stabilizer) and UV stabilizers were incorporated in the matrix systems. So far, the UV stability has been investigated only for blue (spirooxazine) coatings. Fig. 4 shows the dependence of the darkening of coatings containing a blue spirooxazine compared to similar coatings with a HALS 


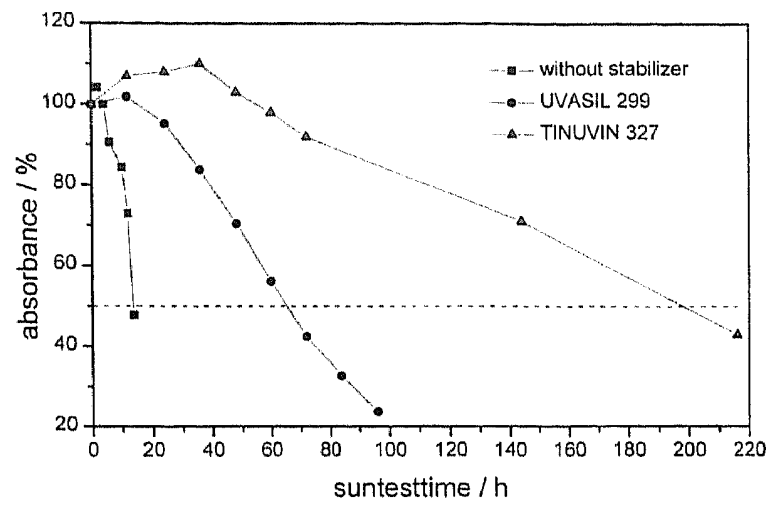

Fig. 4. Darkening $\Delta A_{\| 1}$ of Nanomer ${ }^{\text {in }}$ coatings (with 3 wt. \% $\mathrm{SiO}_{2}$ nanoparticles, see Section 2) containing spirooxazine dye Blue A with Uvasil 299 (HALS). Tinuvin 327 or without any stabilizer during a dry sun-test $(75$ $\mathrm{mW} / \mathrm{cm}^{2}$ ) with an estimated relative error of measurement of $\pm 5 \%$ (the lines are drawn as a guide for the eye).

(Uvasil 299) and with an UV-absorber (Tinuvin 327) on the time in a dry sun-test $\left(75 \mathrm{~mW} / \mathrm{cm}^{2}\right)$.

The half life time (50\% decay of the photochromic effect) of the blue spirooxazine dye incorporated in the coating system in a dry sun-test $\left(75 \mathrm{~mW} / \mathrm{cm}^{2}\right)$ has been increased from $18 \mathrm{~h}$ (without any stabilizer) up to $65 \mathrm{~h}$ by the addition of Uvasil 299 (HALS) and the half life time has been remarkably increased by the addition of Tinuvin 327 (UVabsorber) up to $200 \mathrm{~h}$. It is known from literature [11] that Uvasil 299 acts as a radical scavenger. Tinuvin 327 however acts as a UV absorber for UV light with wavelengths $<340$ $\mathrm{nm}$ [12]. The excitation of the blue dye is obtained with UV light of about $360 \mathrm{~nm}$, as determined by UV-VIS absorbance measurements of an alcoholic dye solution [7]. Therefore it is hypothesized that the absorbance of the 'hard' UV light by Tinuvin 327 offers a more efficient protection than the radical scavenger effect of Uvasil 299.

\section{Conclusion}

The use of a nanocomposite coating matrix with 'hard' and 'soft' microstructural units allows the development of photochromic coatings with sufficient free volume for fast switching dyes in combination with macroscopic hard properties. The development of these coating materials opens promising prospects for applications in the field of ophthalmic and sportive eye protection applications and a broad variety for different decorative applications. Although the feasibility for obtaining long term UV stabilization for the spirooxazine Blue $\mathrm{A}$ by addition of an UV absorber to the coating matrix has been demonstrated, the long term stability of other photochromes will have to be investigated in the future.

\section{References}

[1] H. Dürr, H. Bouas-Laurent (Eds.), Photochromism, Molecules and Systems Elsevier, Amsterdam, 1990, pp. 793, 855, 879.

[2] J.-P. Crano, S. Weber, P. Tardieu, N. Lelard, WO 96/27488 (1996).

[3] L. Hou, B. Hoffmann, M. Mennig, H. Schmidt, Proc. 17th Int. Cong. on Glass: Oct. 9-14 1995 (Beijing) 4 (1995) 234.

[4] V. Malatesta, Photochromic and Thermochromic Compounds (no. 4), Chapter 10.5, 10.9. Plenum Press, New York, 1997.

[5] L. Hou, B. Hoffmann, H. Schmidt, M. Mennig, Sol-Gel Sci, Tech. 8 (1997) 923.

[6] L. Hou, B. Hoffmann, H. Schmidt, M. Mennig, Sol-Gel Sci, Tech. 8 (1997) 927.

[7] M. Lindenstruth, K. Fries, private communication.

[8] L. Hou, M. Mennig, H. Schmidt, Proc. SPIE 2288 (1994) 328.

[9] Din 53151.

[10] H. Schmidt, M. Mennig, G. Jonschker, V. Gerhard, PCT/EP(6/01448, 02.04 .96 .

[11] V. Malatesta, C. Neri, Macromolecules 26 (1993) 4287.

[12] Ciba Geigy product information. 